\title{
16S Ribosomal DNA Sequence Analysis Confirms the Close Relationship between the Genera Xanthobacter, Azorhizobium, and Aquabacter and Reveals a Lack of Phylogenetic Coherence among Xanthobacter Species
}

\author{
FRED A. RAINEY ${ }^{1 *}$ AND J. WIEGEL ${ }^{2}$ \\ DSMZ-Deutsche Sammlung von Mikroorganismen und Zellkulturen GmbH, D-38124 Braunschweig, Germany, ${ }^{1}$ \\ and Department of Microbiology and Center for Biological Resource Recovery, \\ University of Georgia, Athens, Georgia $30602^{2}$
}

\begin{abstract}
A comparative 16S ribosomal DNA (rDNA) sequence analysis was used to investigate the phylogenetic position of members of the genus Xanthobacter. We determined 16S rDNA sequence data for the type strains of the three Xanthobacter species and five additional Xanthobacter strains. The close relationship between the genera Xanthobacter, Azorhizobium, and Aquabacter previously demonstrated by DNA-rRNA hybridization studies was confirmed. The results of our phylogenetic analysis indicate that members of the genera Xanthobacter, Azorhizobium, and Aquabacter are intermixed and that there is no clear generic cluster consisting of the Xanthobacter species. A comparison of the Xanthobacter sequences with the 16S rDNA sequences available from environmental clone studies indicated that members of this genus have not been detected by nonculturing approaches.
\end{abstract}

Phylogenetic analysis based on comparisons of $16 \mathrm{~S}$ ribosomal DNA (rDNA) sequence data is now routinely used in the determination of taxonomic relationships between microorganisms and in the design of taxon-specific probes for use in microbial identification and molecular ecology studies $(1,10$, 15, 21).

One genus for which no full 16S rDNA sequence data are currently available is the genus Xanthobacter. The genus Xanthobacter consists of three species, Xanthobacter agilis (5), Xanthobacter autotrophicus (23), and Xanthobacter flavus (6, 12, 17). These organisms are gram-negative, pleomorphic rods that are able to obtain energy by the oxidation of hydrogen and can fix dinitrogen (22). Xanthobacter species have been isolated from soils, marine and freshwater sediments, the soil of rice fields, and the rhizosphere of rice plants (22).

DNA-rRNA hybridization studies have shown that the three species of the genus Xanthobacter fall within the alpha-2 subclass of the Proteobacteria and are the closest relatives of Aquabacter spiritensis and Azorhizobium caulinodans (2-4).

In order to investigate the relationships of the genera Aquabacter, Azorhizobium, and Xanthobacter, we determined the complete $16 \mathrm{~S}$ rDNA sequences of the type strains of the three Xanthobacter species and additional strains. The $16 \mathrm{~S}$ rDNA sequence of the type strain of Azorhizobium caulinodans was redetermined in order to clarify some ambiguous nucleotide positions in the sequence currently available from the public databases.

The strains were grown on nutrient agar. $16 \mathrm{~S}$ rDNA sequences were determined by directly sequencing the $16 \mathrm{~S}$ rRNA genes amplified by PCR (16). The PCR products were directly sequenced by using a Taq DyeDeoxy terminator cycle sequencing kit (Applied Biosystems, Inc., Foster City, Calif.), and the purified sequence reaction mixtures were electrophoresed by using a model 373A automatic DNA sequencer (Applied Biosystems). The sequences were manually aligned with

\footnotetext{
* Corresponding author. Phone: 49-531-2616100. Fax: 49-531-2616418. Electronic mail address; rainey@gbf-braunschweig.de.
}

the sequences of representatives of the alpha-2 subclass of the Proteobacteria. A phylogenetic analysis was performed by using the "ARB-A Software Environment for Sequence Data" programs (9). The methods of analysis used included distance methods, as well as maximum-likelihood, parsimony, and neighborjoining methods.

The strains whose sequences we determined, their sources, and the nucleotide sequence accession numbers of their $16 \mathrm{~S}$ rDNAs are shown in Table 1.

The 16S rDNA sequences obtained for all of the strains which we studied consisted of 1,410 to 1,414 nucleotides. The 16S rRNA sequence of Azorhizobium caulinodans ORS571 ${ }^{\mathrm{T}}$ (T = type strain) that was available from the Ribosomal Database Project database (11) was found to differ at seven nucleotide positions from the sequence of Azorhizobium caulinodans DSM $5975^{\mathrm{T}}\left(=\mathrm{ORS571^{ \textrm {T } }}\right)$ which we determined. The sequence determined in this study was used in all analyses. The $16 \mathrm{~S}$ rDNA sequence of Aquabacter spiritensis used in the phylogenetic analyses was the sequence available from the Ribosomal Database Project database (11). The results of our phylogenetic analysis based on 16S rDNA sequence comparisons indicated that the genera Aquabacter, Azorhizobium, and Xanthobacter are closely related and represent a distinct cluster within the alpha- 2 subclass of the Proteobacteria. All methods of phylogenetic analysis used in this study resulted in similar trees or dendrograms. In all cases the Aquabacter-Azorhizobium-Xanthobacter cluster of the alpha-2 subclass was recovered with the same internal branching order. Some slight rearrangements in the branching orders of other clusters within the alpha-2 subclass were observed when different methods were used. The phylogenetic dendrogram shown in Fig. 1 was reconstructed by the neighbor-joining method (20) from a distance matrix calculated by using the correction of the algorithm of Jukes and Cantor $(7,14)$.

Figure 1 shows the close relationship among the Xanthobacter species and strains, Aquabacter spiritensis, and Azorhizobium caulinodans. The representatives of the genera Xanthobacter, Aquabacter, and Azorhizobium form a distinct cluster 
TABLE 1. Strains for which $16 \mathrm{~S}$ rDNA sequence data were obtained in this study

\begin{tabular}{llc}
\hline \multicolumn{1}{c}{ Strain } & \multicolumn{1}{c}{ Source } & $\begin{array}{c}\text { Nucleotide sequence } \\
\text { accession no. }\end{array}$ \\
\hline Xanthobacter flavus $301^{\mathrm{T}}$ & J. Wiegel & X94199 \\
Xanthobacter flavus JW/KR-1 & J. Wiegel & X94204 \\
Xanthobacter flavus H4-14 & J. Wiegel & X94202 \\
Xanthobacter flavus JW/KR-E1 & J. Wiegel & X94206 \\
Xanthobacter flavus JW/KR-2 & J. Wiegel & X94205 \\
Xanthobacter agilis SA35 & J. Wiegel & X94198 \\
Xanthobacter autotrophicus $7 C^{\mathrm{T}}$ & J. Wiegel & X94201 \\
Xanthobacter autotrophicus JW33 & J. Wiegel & X94203 \\
Azorhizobium caulinodans ORS571 & DSMZ $^{\mathrm{T}}$ & X94200 \\
\hline
\end{tabular}

${ }^{a}$ DSMZ, Deutsche Sammlung von Mikroorganismen und Zellkulturen $\mathrm{GmbH}$

within the alpha-2 subclass of the Proteobacteria. The distinctness of this cluster was confirmed by the results of a bootstrap analysis in which the cluster was recovered in all of the trees calculated. The close relationship of the species within this group was also demonstrated by the high $16 \mathrm{~S}$ rDNA similarity values, which ranged from 96.7 to $100 \%$ (Table 2).

The three Xanthobacter species represent individual branches within the Aquabacter-Azorhizobium-Xanthobacter cluster, as do Aquabacter spiritensis and Azorhizobium caulinodans. The level of $16 \mathrm{~S}$ rDNA sequence similarity for two strains of $X$. autotrophicus was $99.9 \%$. The same high level of $16 \mathrm{~S}$ rDNA sequence similarity was found with the $X$. flavus strains studied, which included marine isolate H4-14 (8) and wetland rice isolates JW/KR-1, JW/KR-2, and JW/KR-E1 (18, 19). Both the phylogenetic tree and the $16 \mathrm{~S}$ rDNA similarity values show that in some cases the Xanthobacter species are

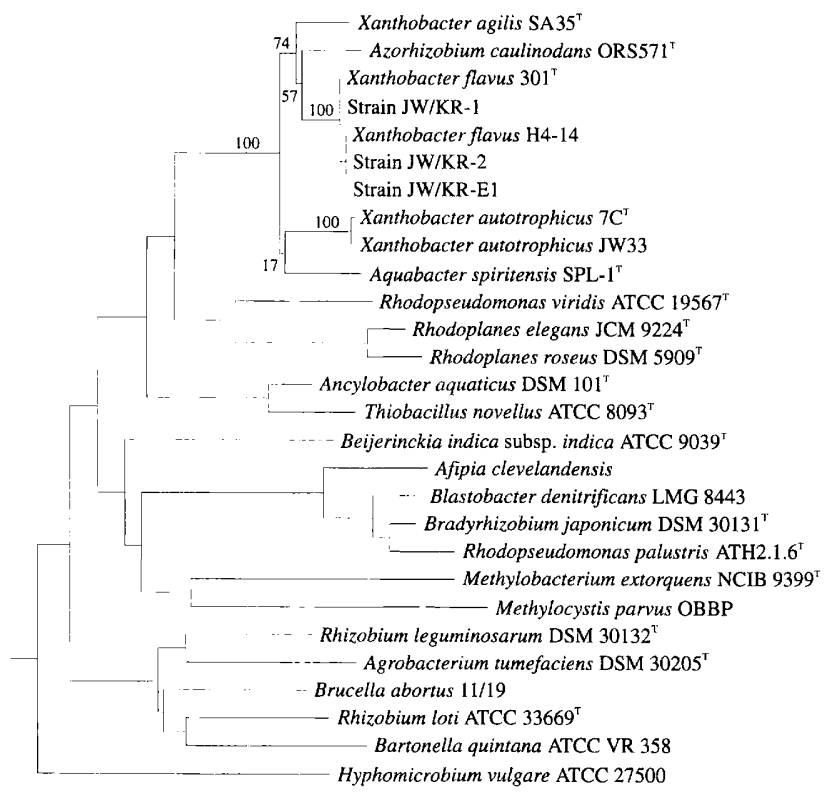

FIG. 1. Phylogenetic dendrogram reconstructed from evolutionary distances $(7,14)$ by the neighbor-joining method $(20)$. Strain designations are included when they were provided by the public databases from which the reference sequences originated. The position of the root was determined by including $E$. coli as an outgroup organism. Scale bar $=5$ inferred nucleotide substitutions pe 100 nucleotides. The numbers given at the nodes represent bootstrap values. equally or more closely related to Aquabacter spiritensis and Azorhizobium caulinodans than they are to each other.

Although we showed that the Aquabacter-AzorhizobiumXanthobacter cluster consists of two subclusters (Fig. 1), this finding is not supported by bootstrap analysis data since the clustering of the two $X$. autotrophicus strains with Aquabacter spiritensis was recovered in only $17 \%$ of the trees constructed. The same is true for the clustering of $X$. agilis, Azorhizobium, and $X$. flavus strains, since this clustering was supported by a bootstrap value of only $74 \%$. The clustering of Azorhizobium caulinodans with the $X$. flavus strains was recovered in only $57 \%$ of the analyses.

In the Ribosomal Database Project release of 17 May 1995 (11) under the section labelled RPS.VIR_SUBGROUP three environmental clone sequences are listed. The Ribosomal Database Project listing indicates that environmental clone sequence MC24 was from an Australian forest soil, while two environmental clone sequences env.PAD53 and env.PAD54 were from rice paddy field soil (11). As this study revealed that the Xanthobacter strains studied are most closely related to the strains in the same Ribosomal Database Project group, these environmental clone sequences were compared with the new Xanthobacter strain sequences determined in this study. Environmental clone sequences env.PAD53 and env.PAD54 consist of only 275 nucleotides (Escherichia coli positions 1115 to 1391), and a comparison showed that they are most similar to the sequences of Rhodoplanes roseus and Rhodoplanes elegans (levels of similarity, 96.9 and $96.2 \%$, respectively) and thus do not fall in the Aquabacter-Azorhizobium-Xanthobacter cluster, to which they are less than $91.5 \%$ similar. The small number of nucleotides available for these environmental clone sequences did not allow an exact phylogenetic position to be determined. Clone MC24 seems to represent a chimeric structure since a comparative analysis showed that the $3^{\prime}$ end of the sequence is similar to the $3^{\prime}$ ends of the sequences of members of the alpha-2 subclass, while the $5^{\prime}$ end shows little similarity to the $5^{\prime}$ ends of the sequences of members of this group. Although Xanthobacter strains have been isolated from rice paddy field soil (18), the two clones recovered from paddy field soil are not representatives of the genus Xanthobacter.

The results of our $16 \mathrm{~S}$ rDNA sequence analysis of the genus Xanthobacter are consistent with the DNA-rRNA hybridization data of Dreyfus et al. (2) and Gillis et al. (3), who showed that the three Xanthobacter species are closely related to Azorhizobium caulinodans. The relatedness of the genera Xanthobacter, Aquabacter, and Azorhizobium previously demonstrated by DNA-rRNA hybridization (2-4) was also confirmed by our $16 \mathrm{~S}$ rDNA sequence analysis. It should be noted that the denaturation temperature of the hybrid of Aquabacter spiritensis DNA and labelled Azorhizobium caulinodans rRNA was $72.9^{\circ} \mathrm{C}(4)$, while the denaturation temperatures of the hybrid of the DNAs of the three Xanthobacter species and Azorhizobium caulinodans rRNA ranged from 77 to $78^{\circ} \mathrm{C}(2,3)$. These data indicate that Aquabacter spiritensis is less closely related to the Xanthobacter species than to Azorhizobium caulinodans, a fact not clearly shown by the $16 \mathrm{~S}$ rDNA sequence analysis.

Our 16S rDNA sequence comparison showed that the three Xanthobacter species are distinct, as was previously established on the basis of DNA-DNA hybridization data (6). Our phylogenetic analysis indicated that the members of the genera Aquabacter, Azorhizobium, Xanthobacter are intermixed and that there is not a clear generic cluster, especially for the Xanthobacter species and strains. The low bootstrap values obtained for some of the internal clusters mean that we should be cautious in interpreting the data and the phylogenetic dendrogram resulting from the data analysis. There are three ways 
TABLE 2. Levels of $16 \mathrm{~S}$ rDNA similarity between strains belonging to the Aquabacter-Azorhizobium-Xanthobacter cluster and related taxa

\begin{tabular}{|c|c|c|c|c|c|c|c|c|c|c|c|c|}
\hline \multirow[b]{2}{*}{ Strain } & \multicolumn{12}{|c|}{$\%$ 16S rDNA similarity } \\
\hline & 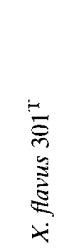 & 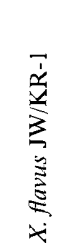 & 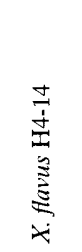 & 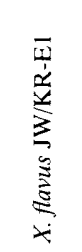 & 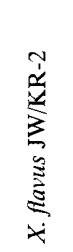 & 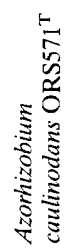 & 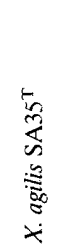 & 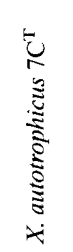 & 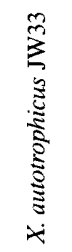 & 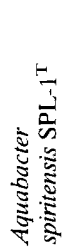 & 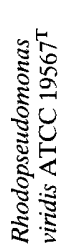 & 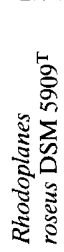 \\
\hline X. flavus JW/KR-1 & 100.0 & & & & & & & & & & & \\
\hline$X$. flavus $\mathrm{H} 4-14$ & 99.9 & 99.9 & & & & & & & & & & \\
\hline$X$. flavus JW/KR-E1 & 99.9 & 99.9 & 100.0 & & & & & & & & & \\
\hline X. flavus JW/KR-2 & 99.9 & 99.9 & 100.0 & 100.0 & & & & & & & & \\
\hline Azorhizobium caulinodans $\mathrm{ORS} 571^{\mathrm{T}}$ & 98.2 & 98.2 & 98.1 & 98.1 & 98.1 & & & & & & & \\
\hline$X$. agilis $\mathrm{SA} 35^{\mathrm{T}}$ & 98.0 & 98.0 & 98.0 & 98.0 & 98.0 & 97.5 & & & & & & \\
\hline$X$, autotrophicus $7 \mathrm{C}^{\mathrm{T}}$ & 97.5 & 97.5 & 97.5 & 97.5 & 97.5 & 96.8 & 96.8 & & & & & \\
\hline$X$. autotrophicus JW33 & 97.5 & 97.5 & 97.4 & 97.4 & 97.4 & 96.7 & 96.7 & 99.9 & & & & \\
\hline Aquabacter spiritensis SPL-1 ${ }^{\mathrm{T}}$ & 97.0 & 97.0 & 96.9 & 96.9 & 96.9 & 96.9 & 97.0 & 97.2 & 97.2 & & & \\
\hline Rhodopseudomonas viridis ATCC $19567^{\mathrm{T}}$ & 92.8 & 92.8 & 92.7 & 92.7 & 92.7 & 93.2 & 92.9 & 92.9 & 92.9 & 93.7 & & \\
\hline Rhodoplanes roseus DSM $5909^{\mathrm{T}}$ & 91.9 & 91.9 & 91.8 & 91.8 & 91.8 & 92.0 & 92.2 & 92.3 & 92.2 & 91.4 & 94.0 & \\
\hline Rhodoplanes elegans JCM $9224^{\mathrm{T}}$ & 92.0 & 92.0 & 91.9 & 91.9 & 91.9 & 92.4 & 92.1 & 92.4 & 92.4 & 91.9 & 94.0 & 98.1 \\
\hline
\end{tabular}

to view or interpret this analysis. First, it is possible that while the $16 \mathrm{~S}$ rDNA sequence data clearly indicate that the three genera are closely related, it is not possible to determine the true intergeneric relationships because of the high levels of sequence similarity, a problem which cannot be resolved by current methods of phylogenetic analysis. Second, the data could be interpreted to show that the genus Xanthobacter is not coherent, as previously published physiological data have suggested (22), and that each of the three Xanthobacter species represents an individual genus. Third, all strains within the Aquabacter-Azorhizobium-Xanthobacter cluster could be viewed as members of one genus, the three genera could be combined, and an emended description of the genus Xanthobacter could be proposed.

In systematic microbiology, it is still thought that descriptions of new taxa should be based on polyphasic data rather than just $16 \mathrm{~S}$ rDNA sequence similarity values (13). The genera Aquabacter, Azorhizobium, and Xanthobacter can be clearly differentiated from each other on the basis of their current descriptions $(2,4,22)$. Aquabacter spiritensis differs from both Azorhizobium caulinodans and Xanthobacter species in that it does not fix nitrogen, contains gas vacuoles, and produces chalky white colonies (4). The stem-nodulating ability of Azorhizobium caulinodans distinguishes this species from all Xanthobacter species (2). Only the species of the genus Xanthobacter have been shown to contain the water-insoluble yellow pigment zeaxathin dirhamnoside (22), although the possible presence of this pigment in members of the other two genera has not been investigated, probably because of the lack of yellow colony pigmentation in these organisms. The data currently available for comparisons of the three Xanthobacter species are morphological and physiological data (22). These data do not include good differential characteristics that would aid in the description of new genera. Therefore, it would be unwise to make taxonomic rearrangements in this group on the basis of our current knowledge. Chemotaxonomic data for these genera should be obtained, and such information may support the differences observed in the phylogenetic analysis.

\section{REFERENCES}

1. Amman, R., W. Ludwig, and K. H. Schleifer. 1993. Identification and in situ detection of individual bacterial cells. FEMS Microbiol. Lett. 100:45-50.
2. Dreyfus, B., J. L. Garcia, and M. Gillis. 1988. Characterization of Azorhizobium caulinodans gen. nov., sp. nov., a stem-nodulating nitrogenfixing bacterium isolated from Sesbania rostrata. Int. J. Syst. Bacteriol. 38:89-98.

3. Gillis, M., J. L. Garcia, and B. Dreyfus. 1989. Phenotypic and genotypic studies on tropical rhizobia leading to the characterization of Azorhizobium caulinodans, p. 19-22. In Proceedings of the International Congress on Sesbania rostrata. Technical Centre for Agricultural and Rural Cooperation ACP/EEC, Wageningen, The Netherlands.

4. Irgens, R. L., K. Kersters, P. Segers, M. Gillis, and J. T. Staley. 1991. Aquabacter spiritensis, gen. nov., sp. nov., an anaerobic, gas-vacuolate aquatic bacterium. Arch. Microbiol. 155:137-142.

5. Jenni, B., and M. Aragno. 1987. Xanthobacter agilis sp. nov., a motile, dinitrogenfixing, hydrogen-oxidizing bacterium. Syst. Appl. Microbiol. 9:254-257.

6. Jenni, B., M. Aragno, and J. K. W. Wiegel. 1987. Numerical analysis and DNA-DNA hybridization studies on Xanthobacter and emendation of Xanthobacter flavus. Syst. Appl. Microbiol. 9:247-253.

7. Jukes, T. H., and C. R. Cantor. 1969. Evolution of protein molecules, p. 21-132. In H. N. Munro (ed.), Mammalian protein metabolism. Academic Press, New York.

8. Lidstrom-O'Connor, M. E., G. L. Fulton, and A. E. Wopat. 1983. "Methylobacterium ethanolicum": a syntrophic association of two methylotrophic bacteria. J. Gen. Microbiol. 129:3139-3148.

9. Ludwig, W. (Technical University, Munich, Germany). 1995. Unpublished data.

10. Ludwig, W., and K. H. Schleifer. 1994. Bacterial phylogeny based on 16S and 23S rRNA sequence analysis. FEMS Microbiol. Rev. 15:155-173.

11. Maidak, B. L., N. Larsen, M. J. McCaughey, R. Overbeek, G. J. Olsen, K. Fogel, J. Blandy, and C. R. Woese. 1994. The Ribosomal Database Project. Nucleic Acids Res. 22:3485-3487.

12. Malik, K. A., and D. Claus. 1979. Xanthobacter flavus, a new species of nitrogen-fixing hydrogen bacteria. Int. J. Syst. Bacteriol. 29:283-287.

13. Murray, R. G. E., D. J. Brenner, R. R. Colwell, P. De Vos, M. Goodfellow, P. A. D. Grimont, N. Pfennig, E. Stackebrandt, and G. A. Zavarzin. 1990. Report of the Ad Hoc Committee on Approaches to Taxonomy within the Proteobacteria. Int. J. Syst. Bacteriol. 40:213-215.

14. Olsen, G. J., R. Overbeek, N. Larsen, T. L. Marsh, M. J. McCaughey, M. A. MacIukenas, W. M. Kaun, T. J. Macke, and C. R. Woese. 1992. The Ribosomal Database Project. Nucleic Acids Res. 20(Suppl.):2199-2200.

15. Olsen, G. J., C. R. Woese, and R. Overbeek. 1994. The winds of (evolutionary) change: breathing new life into microbiology. J. Bacteriol. 176:1-6.

16. Rainey, F. A., M. Dorsch, H. W. Morgan, and E. Stackebrandt. 1992. 16S rDNA analysis of Spirochaeta thermophila: its phylogenetic position and implications for the systematics of the order Spirochaetales. Syst. Appl. Microbiol. 15:197-202.

17. Reding, H. K., C. L. M. Cross, L. Dijkhuizen, and J. Wiegel. 1992. Emendation of Xanthobacter flavus as a motile species. Int. J. Syst. Bacteriol. 42:309-311.

18. Reding, H. K., P. G. Hartel, and J. Wiegel. 1991. Effect of Xanthobacter, isolated and characterized from rice roots, on growth of wetland rice. Plant Soil 138:221-229.

19. Reding, H. K., and J. Wiegel. 1993. Motility and chemotaxis of a Xan- 
thobacter sp. isolated from roots of rice. J. Gen. Microbiol. 139:815-820.

20. Saitou, N., and M. Nei. 1987. The neighbor-joining method: a new method for reconstructing phylogenetic trees. Mol. Biol. Evol. 4:406-425.

21. Ward, N., F. A. Rainey, B. Goebel, and E. Stackebrandt. 1995. Identifying and culturing the 'unculturables'-a challenge for microbiologists, p. 89-110. In D. Allsopp, D. L. Hawksworth, and R. R. Colwell (ed.), Microbial diversity and ecosystem function. CAB International, Wallingford, United Kingdom.
22. Wiegel, J. 1992. The genus Xanthobacter, p. 2365-2383. In A. Balows, H. G. Trüper, M. Dworkin, W. Harder, and K.-H. Schleifer (ed.), The prokaryotes, 2nd ed. Springer-Verlag, New York.

23. Wiegel, J., D. Wilke, J. Baumgarten, R. Opitz, and H. G. Schlegel. 1978. Transfer of the nitrogen-fixing hydrogen bacterium Corynebacterium autotrophicum Baumgarten et al. to Xanthobacter gen. nov. Int. J. Syst. Bacteriol. 28:573-581. 